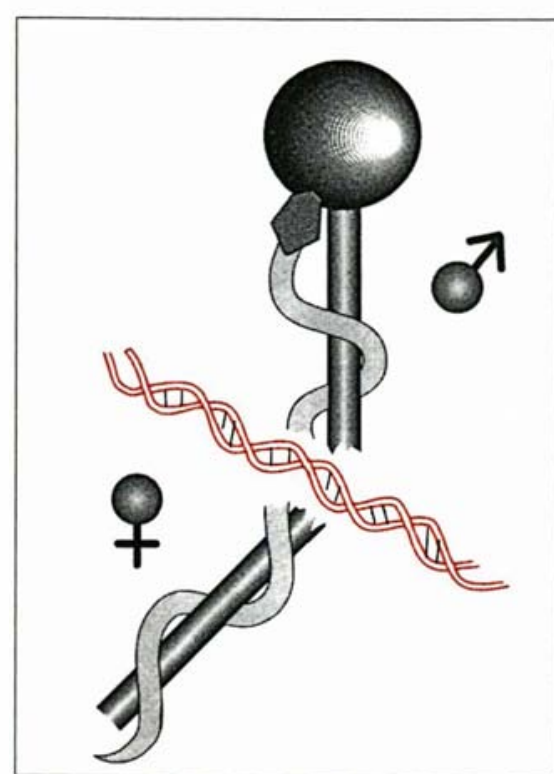

David J. Roy

Centre de bioéthique, Institut de recherches cliniques de Montréal, Faculté de médecine, Université de Montréal, Canada.

TIRÉS A PART

P.J. Roy.

\title{
Refus des traitements de prolongation de vie dans le cas de patients dont la mort n'est pas imminente: éthique clinique et jurisprudence récente au Québec
}

David J. Roy

Les jugements rendus en janvier 1992 par la Cour supérieure du Québec dans les cas de Nancy B. et de Robert Corbeil ont permis de clarifier les limites légales imposées aux médecins et aux institutions de santé en ce qui a trait aux traitements de prolongation de la vie contre la volonté du patient. Selon toute vraisemblance, ces deux décisions influenceront grandement la façon dont les médecins, au Québec et au Canada, résoudront les dilemmes qui se présenteront au chevet du malade.

\section{Contexte éthique et légal}

$\mathrm{Au}$ Québec, tout comme dans les autres provinces canadiennes, ces quinze dernières années ont été marquées par le développement d'un consensus éthique préconisant le respect de la volonté du patient. Dans cette optique, les médecins ont le droit de ne pas entreprendre ou d'interrompre les traitements qui ne font que prolonger l'agonie ou les souffrances du malade. L'expression " permettre aux patients de mourir dans la dignité " rend bien compte de ce nouveau point de vue, que l'on peut résumer ainsi.

- Le principe d'autonomie : les médecins ne sont pas obligés de traiter des patients contre leur volonté, lorsque ceux-ci ont exprimé leur refus de façon lucide, éclairée et continue.
- Le principe de proportionnalité : les médecins ne sont pas obligés d'entreprendre ou de continuer des traitements qui sont thérapeutiquement inutiles ou qui ne sont pas dans le meilleur intérêt du patient.

- Le principe du contrôle de la douleur: il est justifié par les médecins d'utiliser toutes les combinaisons ou les doses d'analgésiques nécessaires pour contrôler la doulcur chez un patient en phase terminale et même si ce faisant, ils devaient hâter la mort.

Ces trois principes représentent actuellement la pensée dominante en éthique et résument trois des principales recommandations de la Commission de réforme du droit du Canada. Ces recommandations, présentées il y a déjà 10 ans, visaient à amender les sections du Code criminel du Canada concernant les devoirs du médecin face à la prolongation de la vie et au soulagement de la souffrance [1].

D'un point de vue éthique, on admet généralement que le principe de proportionnalité autorise à ne pas entreprendre ou à interrompre des traitements de prolongation de vie s'il en résultait une prolongation de l'agonie ou le maintien d'un état végétatif persistant.

Cependant, médecins, infirmières, administrateurs d'hôpitaux et autres travailleurs paramédicaux sont souvent réticents à débrancher le respirateur de patients conscients et lucides, particu- 
lièrement lorsque le pronostic indique qu'ils pourraient vivre encore longtemps. Le cas de Nancy B. illustre bien cette situation. On constate la même réticence à respecter la volonté des patients - des tétraplégiques par exemple - lorsque ceux-ci refusent toute alimentation parce qu'ils désiraient mourir. C'était le cas, en particulier, de Robert Corbeil.

Une telle attitude de la part des professionnels de la santé relève de motivations à la fois éthiques et légales. En effet, les médecins ont tendance à penser qu'en débranchant le respirateur, ils sont en train de tuer un patient qui aurait pu vivre encore assez longtemps. De plus, les craintes des médecins ou des administrateurs d'hôpitaux face aux conséquences légales d'un tel geste n'étaient pas, jusqu'aux récentes décisions de la Cour supérieure du Québec, injustifiées ou sans fondement. En effet, le Code criminel du Canada a été rédigé bien avant que les techniques modernes de prolongation de la vie ne fassent leur apparition.

Dans leur version actuelle, certaines sections de ce même Code placent définitivement le médecin face à un dilemme ; il doit, soit, supposer que la loi actuelle n'a pas le sens qu'on semble lui accorder et pratiquer la médecine dans le meilleur intérêt du patient; soit admettre que la loi est effectivement précise et pratiquer une médecine défensive, afin de minimiser les probabilités de poursuites, même s'il fait fi de l'intérêt du patient [2]. Même si les recommandations de la Commission de réforme du droit du Canada ont été formulées en vue d'amender le Code criminel et, du même coup, dissiper toute incertitude légale, elles n'ont cependant jamais été adoptées. Ces recommandations ont malgré tout influencé la jurisprudence au Canada, comme ce fut le cas au Québec dans les causes de Nancy B. et de Robert Corbeil.

\section{Le cas de Nancy $B$.}

A la fin de 1991 et au début de 1992, le cas de Nancy B. a fait l'objet de nombreux commentaires dans les journaux, à la radio et à la télévision à travers le Canada. Nancy B., une jeune femme de 25 ans atteinte d'une importante atrophie musculaire causée par le syndrome de Guillain-Barré, était confinée au lit depuis plus de deux ans et désirait que le respirateur qui la maintenait en vie fût débranché. Nancy était hospitalisée à l'Hôtel-Dieu de Québec mais, devant la réticence des autorités de cet hôpital à accéder à sa requête, son cas s'est retrouvé devant la Cour supérieure du Québec.

Avant l'apparition de sa maladie, Nancy était une jeune personne très active et en pleine santé. Mais, selon l'opinion des experts, elle ne pouvait espérer aucune amélioration de sa condition et elle serait restée dépendante d'un respirateur pour la vie. Parfaitement lucide et non déprimée pathologiquement, elle demanda alors la cessation de ce traitement de prolongation de vie. La cour fut alors appelée à décider de la requête de Nancy $B$.

Dans sa décision rendue le 6 janvier 1992, le juge Jacques Dufour a clarifié deux points importants de la loi pour appuyer son jugement dans lequel il conclut que la décision de Nancy B. de refuser l'utilisation d'un respirateur devait être respectée. Premièrement, le fait pour un médecin de débrancher un respirateur à la demande libre et éclairée d'un patient ne constitue pas un acte déraisonnable ou un acte de négligence criminelle. Deuxièmement, le débranchement du respirateur dans les circonstances entourant le cas de Nancy B. n'équivaut en aucun cas à un homicide ou à une aide au suicide [3].

Le juge Dufour a ainsi autorisé le médecin traitant de Nancy B., le Dr. Danièle Marceau, à cesser tout support respiratoire à l'heure choisie par Nancy, à la condition d'avoir au préalable vérifié à nouveau cette décision auprès de celle-ci. Nancy est demeurée inébranlable et décéda en février 1992.

\section{Le cas de Robert Corbeil}

M. Corbeil est âgé de 35 ans, marié et père de deux enfants âgés de 9 et 10 ans. Devenu tétraplégique à la suite d'un accident survenu en mai 1990 et atteint de paralysie irréversible, il déclare que la vie ne lui offre aucun intérêt, qu'il ne désire plus vivre à n'importe quel prix et qu'il souhaite mourir. Il refuse donc tout traitement médical et toute nourriture.
Le Manoir de la Pointe-Bleue de Sainte-Marguerite du lac Masson, où il est hébergé, se tourne alors vers les tribunaux afin de déterminer si l'institution et le personnel soignant, plus particulièrement les infirmières et le médecin traitant, doivent respecter la volonté de M. Corbeil.

Dans sa décision, le juge Gontran Rouleau a noté que M. Corbeil demandait à être protégé de toute intervention destinće à l'empêcher de mourir ou à l'obliger de vivre. La question pertinente, selon le juge, était de savoir si M. Corbeil était suffisamment conscient, équilibré et lucide pour prendre une telle décision et qu'il ne s'agissait donc pas de déterminer si cette décision était raisonnable. De plus, seul M. Corbeil pouvait être en mesure de juger de la qualité de sa vie. A la lumière de ces considératioins, le juge Rouleau, dans sa décision du 22 janvier 1992, a statué que l'institution, les infirmières et le médecin traitant se devaient de respecter la liberté et la volonté de M. Corbeil, et qu'on ne devrait pas tenter de lui administrer un traitement contre son gré [4].

Depuis que cette décision a été rendue, M. Corbeil semble avoir repris goût à la vie, il a recommencé à s'alimenter et semble même disposé à se diriger vers des institutions plus spécialisées que le Manoir de la Pointe-Bleue, afin de bénéficier de thérapies de réhabilitation mieux adaptées à ses besoins.

\section{Commentaires}

Les décisions des juges Dufour et Rouleau viennent implicitement sanctionner les recommandations de la Commission de réforme du droit du Canada qui préconisent une modification de certaines sections ambiguës du Code criminel. Après amendement, la loi ne pourrait plus être interprétée comme une obligation pour les médecins de traiter un patient malgré son refus libre et éclairé, ou encore d'initier ou de continuer des traitements thérapeutiques inutiles qui ne sont pas dans le meilleur intérêt du patient.

De plus, ces décisions juridiques confirment et reflètent la réalité quotidienne en éthique clinique dans les hôpitaux du Canada quant aux décisions de prolongation de vie. Une telle 
orientation se distancie de l'éthique rigide visant à prolonger la vie à tout prix et privilégie une éthique avant tout fondée sur la dignité et la qualité de la vie plutôt que sur la préservation de la vie considérée comme valeur absolue.

Les cas Nancy B. et Robert Corbeil illustrent bien le mouvement qui prévaut actuellement en éthique clinique, mouvement qui met avant tout l'accent sur la dignité humaine, laquelle est fortement liéc au pouvoir de chacun de faire respecter ses aspirations et ses désirs les plus chers. Dans cette optique, la requête de Nancy B. suppose implicitement que, pour certaines personnes, la valeur d'un être humain et la qualité de vie se situent bien au-delà du simple concept d'un cerveau conscient et fonctionnel. L'élément crucial est qu'il n'existe aucune loi, qu'elle soit de l'état ou de la moralité, qui puisse justifier l'asservissement de Nancy B. à une machine.

Dans ces jugements, les juges Dufour et Rouleau ont reconnu que la prolongation de la vie à tout prix, particulièrement au prix de souffrances à la limite du supportable pour le patient, s'avère être une attitude plus que déplorable.

\section{Euthanasie : clarification}

Le terme euthanasie, qui tire sa racine étymologique du grec, signifie "belle mort, mort facile et douce ". C'est d'ailleurs ainsi que dans le Pavillon des cancéreux, Soljenitsyne y décrit la mort des vieillards : ils ne combattaient pas la mort mais savaient qu'ils allaient mourir, s'y préparaient avec tranquillité et s'en allaient sereinement, " comme s'ils ne faisaient qu'emménager dans une nouvelle maison" [5]. L'actuel débat médical, éthique, juridique et social sur l'euthanasie, que l'on retrouve jusque devant les tribunaux, dans les médias et au cœur d'innombrables symposiums et publications, est avant tout lié à une réalité incontournable: un très grand nombre de personnes ne meurent pas " comme si elles ne faisaient qu'emménager dans une nouvelle maison ".

De nos jours, le terme euthanasie signifie mettre délibérément fin, rapidement et par souci de compassion, à $m / s n^{\circ} 7$, vol. 8, septembre 92 la vie d'une personne souffrante et mourante. L'acte d'euthanasic, par exemple par injection ou administration orale d'une dose fatale d'une drogue, entraîne habituellement une mort rapide et sans douleur. Un tel acte ne permet pas à la personne de mourir : on met fin à sa vic. D'un autre côté, on permet à un patient de mourir lorsque, dans l'évolution d'une maladie, il n'est plus possible de rétablir la santé, les fonctions ou la conscience de l'individu. Le processus du "mourir" devient alors irréversible.

\section{Conclusion}

Il est difficile de surestimer l'impact que ces récentes décisions auront sur la pratique de l'éthique clinique au Canada. En effet, quand les traitements de prolongation de vie ne font que prolonger le processus du " mourir " ou une condition de vie considérée comme misérable, elles viennent étayer d'une caution légale la distinction du point de vue éthique entre le terme "euthanasie " et l'expression " permettre au patient de mourir". Stopper les traitements de prolongation de vie dans de telles circonstances ne représente pas un acte d'euthanasie, mais plutôt une reconnaissance des limites du pouvoir de la médecine à faire du bien.

Les deux décisions de la Cour supérieure du Québec créent un précédent. Elles reconnaissent que de tels principes peuvent également s'appliquer dans le cas de patients qui, tout en n'étant pas en phase terminale d'une maladie, refusent des traitements de prolongation de vie parce qu'ils considèrent leur condition intolérable.

Face à ce genre de situations, il demeure primordial de ne pas confondre euthanasie et suspension ou interruption de traitements de prolongation de vie. Il existe de sérieuses raisons, qu'il serait trop long d'énumérer ici, pour s'opposer à la légalisation de l'euthanasie. Les décisions de la Cour supérieure du Québec nous démontrent que cesser des traitements considérés comme insupportables par les patients est justifiable et que libérer ces patients de tels traitements, même si la mort en résulte, n'équivaut pas à un acte d'euthanasie

\section{RÉFÉRENCES}

1. Commission de réforme du droit du Canada. Euthanasie, aide au suicide et interruption de traitement. Rapport $n^{\circ} 20$. Ottawa : Ministre des Approvisionnements et Services Canada, 1983 : 33-37.

2. Commission de réforme du droit du Canada, op. cit., 10.

3. Nancy $B(N)$ c. Hôtel-Dieu de Québec, Juge Jacques Dufour, C.S. Québec 200-05-003232-910, 1992-01-06 (J.C. 92-132).

4. Manoir de la Pointe-Bleue (1978) Inc. c. Corbeil. Juge Gontran Roulcau, C.S. T'crrebonne (Saint-Jérôme) 700-05-002743-916, 1992-01-22 (J.C. 92-410).

5. Soljennitsyne A. Le pavillon des cancéreux, Paris : Éditions Édito-Scrvices, 1971. 